\title{
EDITORIAL
}

\section{Operational research in the South Pacific}

\author{
Chris Bullen, ${ }^{1}$ Johan van Griensven ${ }^{2}$
}

http://dx.doi.org/10.5588/pha.14.0034

0 perational research (OR) can be defined in many ways, but in the context of this Journal, Zachariah et al.'s definition - 'the search for knowledge, strategies, or tools that can enhance the quality, effectiveness, or coverage of programmes in which the research is being done' 1 - seems most apt. The eleven articles in this special supplement of Public Health Action highlight the enormous value to be gained using simple OR methods, and, as has been found elsewhere, the latent appetite and opportunity that exists for OR in the Pacific region. ${ }^{1}$ These articles also reveal that the challenges for building sustainable OR capability and capacity in the region should not be underestimated.

Bissell et al. argue that growing OR capability in the region, as the SORT-IT courses have done so effectively to date, will 'get more value from routinely collected data seldom analysed and thereby inform and improve the quality of practice, systems and impact of policies'. ${ }^{2}$ We agree, but with a proviso: capability is one element of success; an equally important consideration is capacity.

The health workforce in the Pacific is thinly spread within and between countries, across a wide range of complex health issues, amply illustrated by the articles in this supplement. They describe studies of tuberculosis, the non-communicable diseases and their risk factors, and the emergence of syndemics ${ }^{3}$ of diabetes and tuberculosis. In many of the smaller Pacific nations, a single individual may have responsibility for clinical, public health and administrative management of all these conditions at a district or even national level. Is it realistic for such individuals to become actively engaged in OR when they return to such a demanding environment after completion of the OR course? Only time will tell.

Bissell et al. recommend, as part of the solution to this challenge, the involvement of regional and local mentors and experts in promoting OR and supporting OR course graduates. ${ }^{2}$ Unquestionably, regional agencies and local academic institutions have a key role. However, there are few of the latter, most are short of experienced researchers, and many are financially fragile. Ongoing partnerships with larger institutions in Australia and New Zealand, with their access to greater resources and availability of expert mentors, are likely to be needed for some time.

However, this model runs the risk of inculcating and perpetuating dependence. Perhaps the greatest challenge, then, for Pacific OR practitioners - and an opportunity for the sustainability of Pacific OR - is to take the helm of the Pacific OR 'canoe' themselves. Local leadership is also vital to the effective communication and translation of OR findings into policy and practice. Who better to become 'knowledge translators' $^{4}$ than local experts, familiar with local culture, language, politics and the other opportunities and obstacles to achieving change in their own setting?

Only under such conditions - ongoing available expert support, balanced with local leadership - can we expect the dual issues of OR capability and capacity to be addressed sufficiently to lead to health improvements for Pacific peoples.

\section{References}

1 Zachariah R, Harries A D, Ishikawa N, et al. Operational research in low-income countries: what, why, and how? Lancet Infect Dis 2009; 9: 711-717.

2 Bissell K, Viney K, Brostrom R, et al. Building operational research capacity in the Pacific. Public Health Action 2014; 4: 000-000.

3 Littleton J, Park J. Tuberculosis and syndemics: implications for Pacific health in New Zealand. Soc Sci Med 2009; 69: 16741680.

4 Pronovost P, Berenholtz S, Needham D. Translating evidence into practice: a model for large scale knowledge translation. BMJ 2008; 337: 963-965.

\author{
AFFILIATIONS \\ 1 National Institute for Health \\ Innovation, The University \\ of Auckland, Auckland, \\ New Zealand \\ 2 Department of Clinical \\ Sciences, Institute of \\ Tropical Medicine, \\ Antwerp, Belgium \\ CORRESPONDENCE \\ e-mails: c.bullen@auckland. \\ ac.nz \\ jvangriensven@itg.be
}

PHA 2014; 4(2): S1 (C) 2014 The Union
Public Health Action (PHA) The voice for operational research. Published by The Union (www.theunion.org), PHA provides a platform to fulfil its mission, 'Health solutions for the poor'. PHA publishes high-quality scientific research that provides new knowledge to improve the accessibility, equity, quality and efficiency of health systems and services.
e-ISSN 2220-8372

Editor-in-Chief: Donald A Enarson, MD, Canada

Contact: pha@theunion.org

PHA website: http://www.theunion.org/index.php/en/journals/pha Article submission: http://mc.manuscriptcentral.com/pha 